\title{
Musical scale estimation for some multiperiodic pulsating stars
}

\author{
B. Ulaș
}

Department of Physics, Onsekiz Mart University of Çanakkale, 17100, Çanakkale, Turkey

\begin{abstract}
The agreement between frequency arrangements of some multiperiodic pulsating stars and musical scales is investigated in this study. The ratios of individual pulsation frequencies of 28 samples of various types of pulsating stars are compared to 57 musical scales by using two different methods. The residual sum of squares of stellar observational frequency ratios is chosen as the indicator of the accordance. The result shows that the arrangements of pulsation frequencies of Y Cam and HD 105458 are similar to Diminished Whole Tone Scale and Arabian(b) Scale, respectively.
\end{abstract}

Individual Objects: Y Cam, HD 105458

\section{Introduction}

Musical scales are made of several musical notes. Since every note is defined by a typical definite frequency value, it is possible to say that a musical scale is a group of frequencies, listed from the low frequency to high, in one octave. Although every scale has its mathematical representation, their creation and evolution strongly depend on social and cultural structure of the societies as well as the traditional musical instruments.

Multiperiodic pulsating stars show more than one pulsation frequency. They are generally classified according to their pulsational behaviours, shape of light curves and pulsation periods. The frequencies can be obtained by using photometric or spectroscopic methods and analyses. These analyses provide information about the interiors of the stars which plays an important role in astrophysics.

To investigate the agreement between frequency arrangements of some multiperiodic pulsating stars and musical scales, we first arranged the stellar frequencies from low to high frequency in order to make the investigation meaningful and the data comparable. Then we had two data sets - one for musical scale and one for the star - so as to apply the process. The residual sum of squares (RSS) of stellar observational frequencies from the musical ones was chosen as the indicator of the agreement. The probability $(\mathrm{P})$ yielded from the $t$-test for these two data sets was also calculated (Salkind 2006). We compared 57 musical scales (Slonimsky 1986, Berle 1997) which can be classified in 9 scale groups ${ }^{1}$ (Bebop, Blues, Exotic, Harmonic, Major, Melodic Minor, Pentatonic, Symmetric, other scales) to 28 pulsating stars of $\beta$ Cep, $\delta$ Sct, $\gamma$ Dor, oEA, roAp, RV Tau, sdB, solar-like, SPB, and ZZ Cet type ${ }^{1}$.

${ }^{1}$ The complete list of musical scales and stars used in this study is available online from http://bulash.googlepages.com/list 
Table 1: The results of the calculation for Y Cam (oEA type) and HD 105458 ( $\gamma$ Dor type). S1 and S2 refer two different calculation types described in the text. The sample song given in the last column uses estimated scale - partly or completely.

\begin{tabular}{clcccc}
\hline Calculation & Star & Scale & RSS & P & Sample \\
\hline S1 & Y Cam & Diminished Whole Tone & 0.003 & 0.861 & 1 \\
& HD 105458 & Lydian & 0.007 & 0.974 & 2 \\
\hline S2 & Y Cam & Spanish & 0.005 & 0.689 & 3 \\
& HD 105458 & Arabian & 0.003 & 0.776 & 4 \\
\hline
\end{tabular}

1 Antonio C. Jobim - The Girl from Ipanema

2 Joe Satriani - Flying in a Blue Dream

3 Pink Floyd - Matilda Mother

4 Nikolas Roubanis - Misirlou
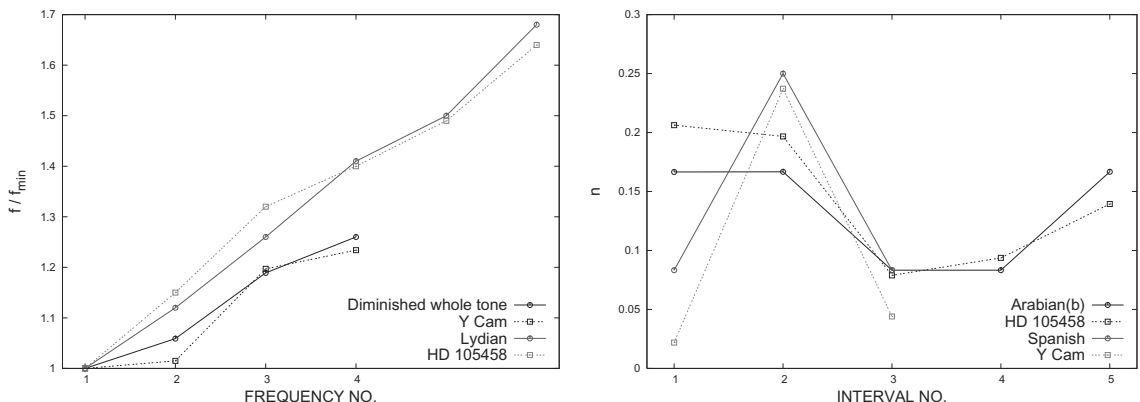

Figure 1: The best RSS value is reached for Y Cam (left) and HD 105458 (right) with the Diminished Whole Tone Scale and Arabian(b) Scale, respectively.

\section{Calculations}

Musical scales are built based on the ratios of frequencies that the human ear is sensitive to. The simple fractions, e.g., 2:1, 3:2, 4:3 are said to be consonant. We first calculated the ratio of the frequency of a given note to the lowest frequency of the scale. For instance, the Blues Scale has the frequency ratios $1-1.19-1.33-1.41-1.50-1.78-2$. Accordingly, as an additional example, the same sequence for a star, say HD 209295 (Handler et al. 2002), is $1-1.03-1.56-1.99-2.03-2.28$, from the low frequency to high. The best results of the calculation are shown in S1 part of Table 1 and plotted in Fig. 1.

Additionally, we compared the intervals which are ratios between consecutive frequencies. The formula is $n=\log _{2}\left(f_{i+1} / f_{i}\right)$ (Wood 2007), where $n$ and $f$ refer to the interval and the frequency value, respectively. The indices, $i$ and $i+1$, denote two consecutive frequencies, for example consonant tones: $n=0$ for unison $(1: 1), n=1$ for octave $(2: 1)$, and $n=0.58$ for fifth $(3: 2)$. The Arabian(b) Scale has intervals for the first six notes $0.17-0.17-0.08-0.08-0.17$, while these for the star HD 105458 (Henry et al. 2001) are $0.21-0.19-0.08-0.09-0.14$. The results are listed in S2 part of Table 1 and plotted in Fig. 1. The numbers in the x-axis represent the location of the frequencies used, e.g. 1 denotes the interval of the first and the second frequencies of the group, while 2 denotes the same for second and third one. 


\section{Conclusion}

The best RSS value is 0.003 for $Y$ Cam with Diminished Whole Tone Scale estimation in the first calculation while the investigation of intervals resulted in the same value for HD 105458 with the Arabian(b) Scale estimation. Some stars can be estimated by more than one scale because of the fact that some scales contain the same notes in their first four or five orders.

\section{References}

Berle, A. 1997, Mel Bay's Encyclopedia of Scales, Modes and Melodic Patterns, Pacific, Mel Bay Publ. Handler, G., Balona, L. A., Shobbrook, R. R., et al. 2002, MNRAS, 333, 262

Henry, G. W., Fekel, F. C., Kaye, A. B., et al. 2001, AJ, 122, 3383

Salkind, J. 2006, Statistics for People Who (think they) Hate Statistics, California, Sage Publications Slonimsky, N. 1986, Thesaurus Of Scales And Melodic Patterns, New York, Macmillan Publishing Co Wood, A. 2007, The Physics Of Music, Davies Press

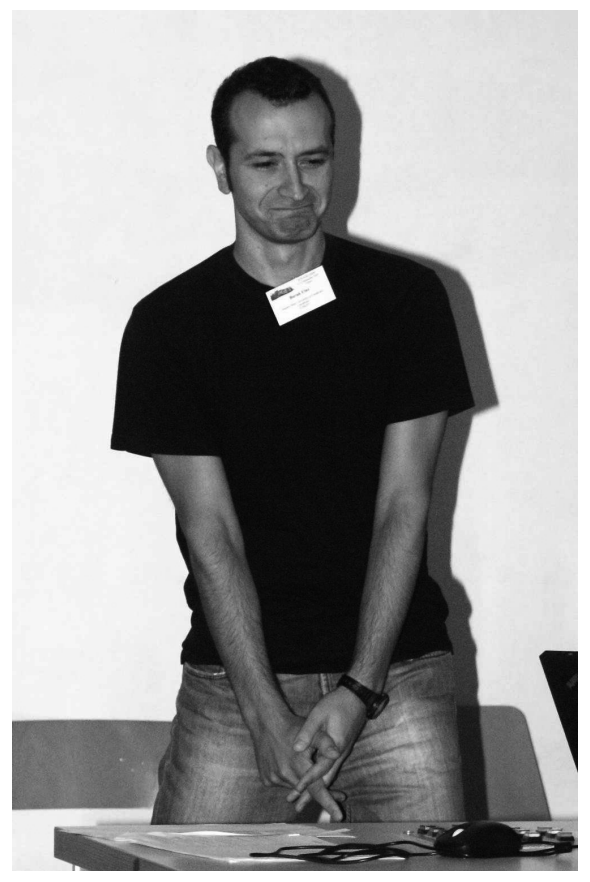



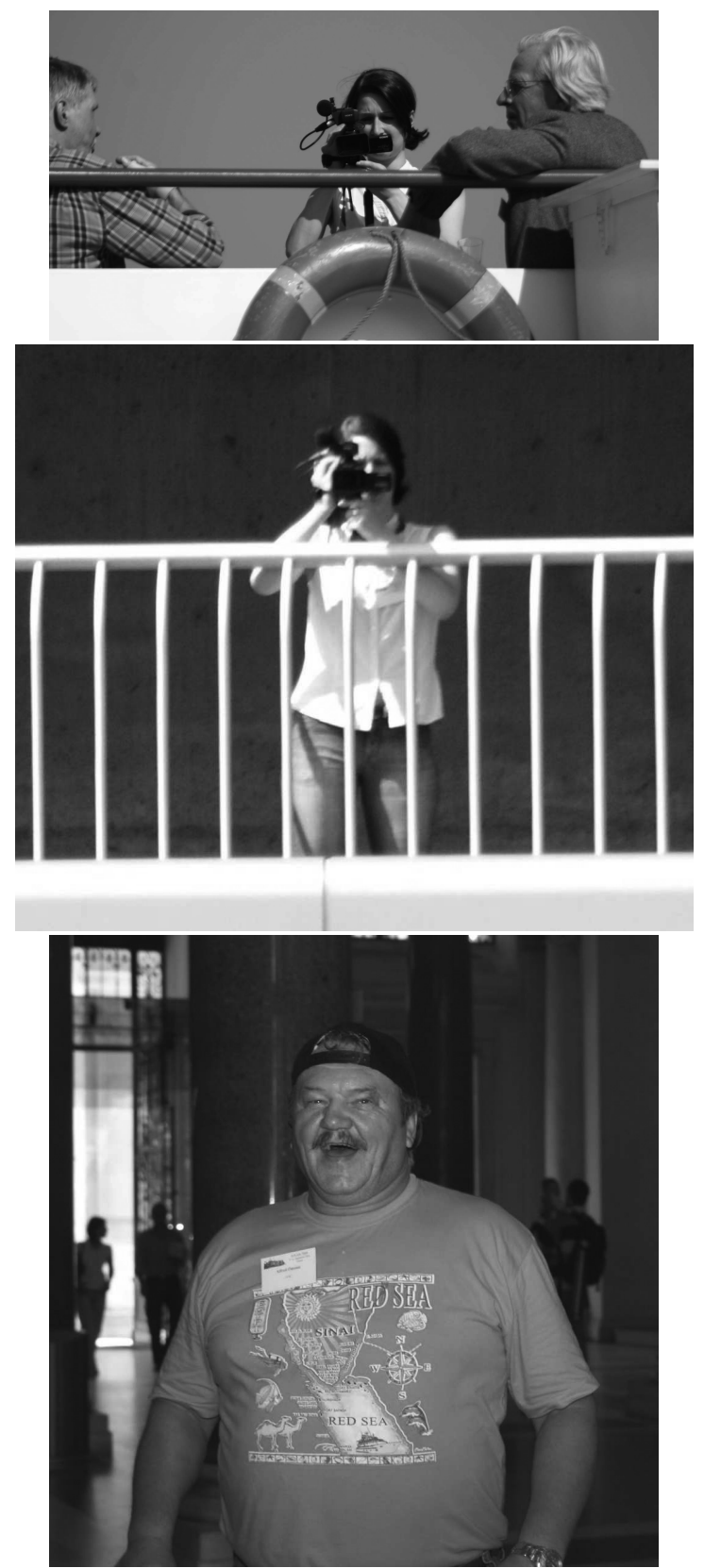

Special thanks to the photographers who kindly provided the snapshots included in this volume: Denise Lorenz and Alfred Omann. 